ANNALES

POLONICI MATHEMATICI

$81.2(2003)$

\title{
Théorèmes de préparation Gevrey et étude de certaines applications formelles
}

\author{
par Augustin Mouze (Lille)
}

\begin{abstract}
We consider subrings $A$ of the ring of formal power series. They are defined by growth conditions on coefficients such as, for instance, Gevrey conditions. We prove preparation theorems of Malgrange type in these rings. As a consequence we study maps $F$ from $\mathbb{C}^{s}$ to $\mathbb{C}^{p}$ without constant term such that the rank of the Jacobian matrix of $F$ is equal to 1 . Let $\mathcal{A}$ be a formal power series. If $F$ is a holomorphic map, the following result is well known: $\mathcal{A} \circ F$ is analytic implies there exists a convergent power series $\widetilde{\mathcal{A}}$ such that $\mathcal{A} \circ F=\widetilde{\mathcal{A}} \circ F$. We get similar results when the map $F$ is no longer holomorphic.
\end{abstract}

Introduction. Soit $X=\left(X_{1}, \ldots, X_{s}\right)$ des variables. On désigne par $\mathbb{C}[[X]]$ l'anneau des séries formelles en $s$ variables à coefficients dans le corps $\mathbb{C}$. Pour tout multi-indice $J$ de $\mathbb{N}^{s}$, on utilise les notations habituelles $X^{J}=$ $X_{1}^{j_{1}} \ldots X_{s}^{j_{s}}$ et $|J|=j_{1}+\ldots+j_{s}$. Pour toute série $f=\sum_{J \in \mathbb{N}^{s}} f_{J} X^{J}$, on note $\operatorname{ord}(f)=\min \left(j \in \mathbb{N}: j=|J|\right.$ et $\left.f_{J} \neq 0\right)$, l'ordre d'annulation de $f$, avec la convention ord $(f)=\infty$ si $f$ est identiquement nul. On considère $M=\left\{M_{n}\right\}_{n \in \mathbb{N}}$ une suite de réels positifs qui vérifie les propriétés

$$
\begin{gathered}
M_{0}=1 \text { et }\left\{M_{n+1} / M_{n}\right\}_{n \in \mathbb{N}} \text { est croissante, } \\
\exists C>0 \text { tel que } M_{n+1} \leq C^{n+1} M_{n} .
\end{gathered}
$$

Dans la suite, pour tout entier fixé $k, M^{(k)}$ désigne la suite $\left\{M_{k n}\right\}_{n \in \mathbb{N}}$. Soit $C_{0}$ une constante strictement positive. On pose

$$
\mathbb{C}[[X]](M)=\bigcup_{C_{0}>0}\left\{f=\sum_{J \in \mathbb{N}^{s}} f_{J} X^{J} \in \mathbb{C}[[X]]: \sup _{J \in \mathbb{N}^{s}} \frac{\left|f_{J}\right|}{M_{|J|} C_{0}^{|J|}}<\infty\right\} .
$$

La condition $\left(\mathrm{H}_{1}\right)$ sur la suite $M$ assure la stabilité par produit de $\mathbb{C}[[X]](M)$. C'est donc un anneau. Pour rappeler qu'il est défini par des conditions de croissance sur les coefficients, on dit que $\mathbb{C}[[X]](M)$ est un anneau de séries

2000 Mathematics Subject Classification: 13F25, 32B05, 14B12, 13 J05.

Key words and phrases: preparation theorems, Gevrey series, local analytic geometry. 
formelles à croissance contrôlée. Si l'on a $M_{n}=1$ pour tout entier $n$, on note $M=\mathbf{1}$ et $\mathbb{C}[[X]](\mathbf{1})$ n'est autre que $\mathbb{C}\{X\}$, l'anneau des séries convergentes. On a l'inclusion $\mathbb{C}\{X\} \subset \mathbb{C}[[X]](M) \subset \mathbb{C}[[X]]$. La condition $\left(\mathrm{H}_{2}\right)$ assure simplement la stabilité par dérivation de $\mathbb{C}[[X]](M)$. Des exemples de tels anneaux sont donnés par les séries formelles à croissance Gevrey $\left(M_{n}=n !^{\alpha}\right.$, $\alpha \in \mathbb{R}_{+}$) bien connues dans la théorie des équations différentielles à coefficients polynômiaux [7]. De plus, $\mathbb{C}[[X]](M)$ est stable par inversion, ce qui permet d'affirmer que $\mathbb{C}[[X]](M)$ est un anneau local d'idéal maximal $\underline{m}_{X, M}$ l'ensemble des séries de $\mathbb{C}[[X]](M)$ sans terme constant. On note aussi $\underline{m}_{X}$ l'idéal maximal de l'anneau $\mathbb{C}[[X]]$. On sait que $\mathbb{C}[[X]](M)$ est un anneau noethérien [2]. C'est une conséquence de la version plus restrictive du théorème de division de Weierstrass dans $\mathbb{C}[[X]](M)$ établie par J. Chaumat et A.-M. Chollet [2] (l'énoncé classique met en évidence une perte de régularité sur la croissance des coefficients du quotient et du reste). Dans le même esprit, des théorèmes de division du type Hironaka par plusieurs séries ont été établis dans $\mathbb{C}[[X]](M)$ (cf. [10]). C'est le point de départ de ce travail. Les principaux énoncés sont repris en préliminaires.

Dans la première partie, on applique ces théorèmes de division pour obtenir un théorème de préparation du type Malgrange $[8]$ dans $\mathbb{C}[[X]](M)$. C'est le théorème 2.

ThÉORÈme. Soient $F_{1}, \ldots, F_{k}$ des éléments de $\mathbb{C}[[X]](M)$. On note $\mathcal{I}_{M}$ l'idéal engendré par ces éléments sur $\mathbb{C}[[X]](M)$. On suppose que la dimension de l'espace vectoriel $\mathbb{C}[[X]](M) / \mathcal{I}_{M}$, notée $\mu$, est finie et non nulle. Soient $e_{1}, \ldots, e_{\mu}$ des monômes tels que leurs classes d'équivalence dans $\mathbb{C}[[X]](M) / \mathcal{I}_{M}$ forment une base de l'espace vectoriel $\mathbb{C}[[X]](M) / \mathcal{I}_{M}$. Alors, pour tout élément $g(X)$ de $\mathbb{C}[[X]](M)$, il existe des séries $R_{i}\left(u_{1}, \ldots, u_{k}\right)$, avec $1 \leq i \leq \mu$, de $\mathbb{C}[[u]]\left(M^{(\mu)}\right)$ telles que l'on ait

$$
g(X)=\sum_{i=1}^{\mu} R_{i}\left(F_{1}, \ldots, F_{k}\right) e_{i}(X) .
$$

Dans le cas des fonctions ultradifférentiables, des résultats semblables ont été donnés par J. Chaumat et A.-M. Chollet [3]. L'auteur montre ici, que, quitte à bien choisir des générateurs $\Phi_{1}, \ldots, \Phi_{p}$ de l'idéal $\mathcal{I}_{M}$, on a, pour tout $g$ de $\mathbb{C}[[X]](M)$, une décomposition

$$
g(X)=\sum_{i=1}^{\mu} S_{i}\left(\Phi_{1}, \ldots, \Phi_{p}\right) e_{i}(X)
$$

avec, pour tout $i=1, \ldots, p$, l'appartenance de $S_{i}\left(\Phi_{1}, \ldots, \Phi_{p}\right)$ à $\mathbb{C}[[X]](M)$. C'est le théorème 4 . L'exemple 5 montre qu'il est indispensable de bien présenter l'idéal pour obtenir l'information supplémentaire, après recomposition, donnée par le théorème 4 . 
Dans la deuxième partie, en utilisant judicieusement ces théorèmes de préparation, on généralise à $\mathbb{C}[[X]](M)$ un théorème établi par $\mathrm{A}$. Płoski $[14]$ sur les dérivations analytiques. On note $\operatorname{Der}(\mathbb{C}[[X]](M))$ le $\mathbb{C}[[X]](M)$ module des dérivations de l'anneau $\mathbb{C}[[X]](M)$ dans $\mathbb{C}[[X]](M)$. Pour tout idéal $\mathcal{I}_{M}$ de $\mathbb{C}[[X]](M)$, on pose $\operatorname{Der}\left(\mathcal{I}_{M}\right)=\left\{D \in \operatorname{Der}(\mathbb{C}[[X]](M)): D\left(\mathcal{I}_{M}\right)\right.$ $\subset \mathcal{I}_{M}$ \}. On a alors le résultat suivant. C'est le théorème 7 .

ThÉorème. Soit $\mathcal{I}_{M}$ un idéal premier de $\mathbb{C}[[X]](M)$. Soient $f_{1}$ et $f_{2}$ des éléments de $\underline{m}_{X, M}$. On suppose que, pour toutes dérivations $D, D^{\prime}$ de $\operatorname{Der}\left(\mathcal{I}_{M}\right)$, la série $D f D^{\prime} g-D^{\prime} g D f$ appartient à $\mathcal{I}_{M}$. Alors il existe un entier $d$ et une série $P(u, v) \neq 0$ de $\mathbb{C}[[u, v]]\left(M^{(d)}\right)$ tels que $P\left(f_{1}, f_{2}\right)$ appartient $\grave{a} \mathcal{I}_{M}$.

La preuve s'inspire de celle établie par A. Płoski dans le cas analytique [14]. Cependant les théorèmes classiques de préparation et de division de Weierstrass n'étant plus vrais, il faut contourner cette difficulté.

Dans la suite, on considère des applications du type $F(X)=\left(f_{1}(X), \ldots\right.$, $\left.f_{p}(X)\right)$ de $\mathbb{C}^{s}$ dans $\mathbb{C}^{p}$ avec, pour $i=1, \ldots, p, f_{i}$ dans $\mathbb{C}[[X]](M), f_{i}(0)=0$ et telles que la matrice jacobienne de l'application $F$ soit de rang 1 . On note $\mathcal{R}$ l'idéal des relations entre les $f_{i}, 1 \leq i \leq p$, défini par

$$
\mathcal{R}=\left\{R\left(u_{1}, \ldots, u_{p}\right) \in \mathbb{C}[[u]]: R\left(f_{1}, \ldots, f_{p}\right)=0\right\} .
$$

Lorsque $M=\mathbf{1}$, c'est-à-dire lorsque $F$ est à composantes analytiques, on sait que $\mathcal{R}$ est non nul et est engendré par des séries elles-aussi analytiques. C'est un cas particulier du théorème d'A. M. Gabrielov [6]. À l'aide du théorème précédent, on généralise ce résultat pour une suite $M$ quelconque. On montre alors que $\mathcal{R}$ est engendré par des séries de $\mathbb{C}[[u]]\left(M^{(d)}\right)$, avec $d=\min \left(\operatorname{ord}\left(f_{i}\right)\right)$. C'est la proposition 14 .

Enfin, on résout un problème de composition. On sait, d'après $\left(\mathrm{H}_{1}\right)$, que, si $\mathcal{A}$ et $g_{1}, \ldots, g_{s}$ sont des séries de $\mathbb{C}[[X]](M)$, alors $\mathcal{A}\left(g_{1}, \ldots, g_{s}\right)$ appartient encore à $\mathbb{C}[[X]](M)$. Réciproquement, si $G=\left(g_{1}, \ldots, g_{s}\right)$ est une application submersive, on a (cf. [11])

(*) $\quad$ il existe un entier $d$ tel que, pour tout $\mathcal{A}$ de $\mathbb{C}[[X]]$, si $\mathcal{A} \circ G$ appartient à $\mathbb{C}[[X]](M)$ alors $\mathcal{A}$ appartient à $\mathbb{C}[[X]]\left(M^{(d)}\right)$.

C'est une généralisation du cas analytique [5]. On peut également consulter [4] pour le cas mixte.

On établit ici le résultat suivant.

ThÉORÈme. Soit $M$ une suite de réels positifs vérifiant $\left(\mathrm{H}_{1}\right)$ et $\left(\mathrm{H}_{2}\right)$. Soit une application $F(X)=\left(f_{1}(X), \ldots, f_{p}(X)\right)$ de $\mathbb{C}^{s}$ dans $\mathbb{C}^{p}$ avec, pour tout $1 \leq i \leq p, f_{i}(X)$ dans $\mathbb{C}[[X]](M)$. On note $\mathcal{J}_{F}$ sa matrice jacobienne. On suppose $\operatorname{rang}\left(\mathcal{J}_{F}\right)=1$. Alors 
(**) il existe un entier d tel que, pour tout $\mathcal{A}$ de $\mathbb{C}[[X]]$, si $\mathcal{A} \circ F$ appartient $\grave{a} \mathbb{C}[[X]](M)$, il existe une série $\widetilde{\mathcal{A}}$ de $\mathbb{C}[[X]]\left(M^{(d)}\right)$ telle que $\widetilde{\mathcal{A}} \circ F=$ $\mathcal{A} \circ F$.

De plus, l'entier $d=\min \left(\operatorname{ord}\left(f_{i}\right)\right)$ convient.

C'est le théorème 16. C'est là encore une généralisation d'un cas particulier du théorème de A. M. Gabrielov dans le cas analytique [6].

Préliminaires : théorèmes de division dans $\mathbb{C}[[X]](M)$. Dans toute la suite, pour tout $f$ de $\mathbb{C}[[X]]$, on note $f_{J}$ l'élément de $\mathbb{C}$ défini par la formule $f(X)=\sum_{J \in \mathbb{N}^{s}} f_{J} X^{J}$. On définit alors le support de $f$ par

$$
\operatorname{Supp}(f)=\left\{J \in \mathbb{N}^{s}: f_{J} \neq 0\right\} .
$$

On ordonne l'ensemble des multi-indices $J$ de $\mathbb{N}^{s}$ par l'ordre lexicographique de $\left(|J|, j_{1}, \ldots, j_{s}\right)$. On appelle alors bon exposant privilégié de $f$ le multiindice $\operatorname{Exp}(f)$ de $\mathbb{N}^{s}$ défini par

$$
\operatorname{Exp}(f)=\min \left(J \in \mathbb{N}^{s}: J \in \operatorname{Supp}(f)\right) .
$$

Soient $p$ multi-indices de $\mathbb{N}^{s}, E_{1}, \ldots, E_{p}$. On note

$$
\Delta=\bigcup_{i=1}^{p}\left(E_{i}+\mathbb{N}^{s}\right) .
$$

On choisit alors une partition $\Delta=\Delta_{1} \cup \ldots \cup \Delta_{p}$ de $\Delta$ telle que, pour tout $i=1, \ldots, p, \Delta_{i} \subset E_{i}+\mathbb{N}^{s}$. Par exemple, on pose $\Delta_{1}=E_{1}+\mathbb{N}^{s}$, $\Delta_{2}=\left(E_{2}+\mathbb{N}^{s}\right)-\Delta_{1}, \ldots, \Delta_{i}=\left(E_{i}+\mathbb{N}^{s}\right)-\left(\Delta_{1} \cup \ldots \cup \Delta_{i-1}\right)$. Dans toute la suite, on dira que $\mathbb{N}^{s}=\Delta_{1} \cup \ldots \cup \Delta_{p} \cup\left(\mathbb{N}^{s}-\Delta\right)$ est la partition de $\mathbb{N}^{s}$ associée aux $E_{i}$, pour $i=1, \ldots, p$.

Dans [10], on trouve alors le résultat suivant que l'on utilisera tout au long de ce travail.

ThÉORÈme A $([10])$. Soient $f_{1}, \ldots, f_{p}$ dans $\mathbb{C}[[X]](M)$. Pour tout $i=$ $1, \ldots, p$, on pose $E_{i}=\operatorname{Exp}\left(f_{i}\right)$. Alors, pour tout élément $g$ de $\mathbb{C}[[X]](M)$, il existe des uniques séries $g_{1}, \ldots, g_{p}, h$ de $\mathbb{C}[[X]](M)$ telles que l'on ait

$$
\begin{gathered}
g=\sum_{i=1}^{p} f_{i} h_{i}+h_{0}, \\
\operatorname{Supp}\left(h_{i}(X) X^{E_{i}}\right) \subset \Delta_{i}, \quad i=1, \ldots, p, \\
h_{0}=\sum_{b=0}^{\infty} \sum_{B \in \mathbb{N}^{s}} h_{B}^{0} X^{B} \quad \text { avec } h_{B}^{0}=0 \text { pour } B \in \Delta,
\end{gathered}
$$

où $\mathbb{N}^{s}=\Delta_{1} \cup \ldots \cup \Delta_{p} \cup\left(\mathbb{N}^{s}-\Delta\right)$ est la partition de $\mathbb{N}^{s}$ associée aux $E_{i}$, $1 \leq i \leq p$. 
Dans le cas où $p=1$ et $E_{1}=(i, 0, \ldots, 0)$, le théorème $\mathrm{A}$ n'est autre que la version du théorème de division de Weierstrass dans $\mathbb{C}[[X]](M)$ (cf. $[2])$.

Étant donné un idéal $\mathcal{I}_{M}$ de $\mathbb{C}[[X]](M)$, on note $\operatorname{Exp}(\mathcal{I})$ l'ensemble des bons exposants privilégiés des éléments de $\mathcal{I}_{M}$. On appelle alors bonne base standard de $\mathcal{I}_{M}$ une famille $\left(g_{1}, \ldots, g_{k}\right)$ de $\mathcal{I}_{M}$ telle que, si l'on note pour tout $i=1, \ldots, k, E_{i}=\operatorname{Exp}\left(g_{i}\right)$, on ait $\operatorname{Exp}(\mathcal{I})=\bigcup_{i=1}^{k}\left(E_{i}+\mathbb{N}^{s}\right)$. On a aussi le théorème suivant.

ThÉorème B $([10])$. Soient $f_{1}, \ldots, f_{p}$ des éléments de $\mathbb{C}[[X]](M)$. On note $\mathcal{I}_{M}$ l'idéal engendré par ces éléments sur $\mathbb{C}[[X]](M)$. Il existe alors une bonne base standard $G=\left(g_{1}, \ldots, g_{k}\right)$ pour $\mathcal{I}_{M}$ telle que, pour tout $i=$ $1, \ldots, k, g_{i}$ appartienne $\mathbb{C}[[X]](M)$, et telle que, pour tout $g$ dans $\mathbb{C}[[X]](M)$, on puisse écrire $g=\sum_{i=1}^{k} h_{i} g_{i}+h_{0}$ avec

$$
\begin{gathered}
h_{i} \in \mathbb{C}[[X]](M), \quad i=0, \ldots, k, \\
\operatorname{Supp}\left(h_{i}(X) X^{E_{i}}\right) \subset \Delta_{i}, \quad i=1, \ldots, k, \\
h_{0}=\sum_{b=0}^{\infty} \sum_{\substack{B \in \mathbb{N}^{s} \\
|B|=b}} h_{B}^{0} X^{B} \quad \text { avec } h_{B}^{0}=0 \text { pour } B \in \Delta,
\end{gathered}
$$

où $\mathbb{N}^{s}=\Delta_{1} \cup \ldots \cup \Delta_{k} \cup\left(\mathbb{N}^{s}-\Delta\right)$ est la partition de $\mathbb{N}^{s}$ associée aux $E_{i}$, $1 \leq i \leq k$. De plus, g appartient à $\mathcal{I}_{M}$ si et seulement si $h_{0}=0$.

\section{Deux théorèmes de préparation}

1. Définitions et notations. Soit $M$ une suite de réels positifs vérifiant les conditions $\left(\mathrm{H}_{1}\right)$ et $\left(\mathrm{H}_{2}\right)$. Soit $F=\left(F_{1}(X), \ldots, F_{k}(X)\right)$ un élément de $(\mathbb{C}[[X]](M))^{k}$. On note $\mathcal{I}_{M}$ l'idéal engendré par les séries $F_{1}(X), \ldots, F_{k}(X)$ sur $\mathbb{C}[[X]](M)$. On suppose que

le $\mathbb{C}$-espace vectoriel $\mathbb{C}[[X]](M) / \mathcal{I}_{M}$ est de dimension finie $\mu$, avec $\mu \geq 1$.

Ceci implique $F(0)=0$ et $k \geq s$. On note $e_{1}, \ldots, e_{\mu}$ des monômes tels que leurs classes d'équivalence dans $\mathbb{C}[[X]](M) / \mathcal{I}_{M}$ forment une base de l'espace vectoriel $\mathbb{C}[[X]](M) / \mathcal{I}_{M}$. On associe à $F_{1}, \ldots, F_{k}$ une bonne base standard $\Phi=\left(\Phi_{1}, \ldots, \Phi_{p}\right)$ de l'idéal $\mathcal{I}_{M}$. On note alors, pour tout $i=1, \ldots, p, E_{i}$ le bon exposant privilégié de $\Phi_{i}$. On pose $\Delta=\bigcup_{i=1}^{p}\left(E_{i}+\mathbb{N}^{s}\right)$. Comme le $\mathbb{C}$-espace vectoriel $\mathbb{C}[[X]](M) / \mathcal{I}_{M}$ est de dimension finie $\mu$, l'ensemble $\mathbb{N}^{s}-\Delta$ a exactement $\mu$ éléments. On note $m_{1}(X), \ldots, m_{\mu}(X)$ les monômes associés. Leurs classes d'équivalence modulo $\mathcal{I}_{M}$ engendrent donc $\mathbb{C}[[X]](M) / \mathcal{I}_{M}$.

On se propose d'établir des théorèmes qui rappellent dans leur forme le théorème classique de préparation de Malgrange [8].

2. ThÉorème. Soient $F_{1}, \ldots, F_{k}$ des éléments de $\mathbb{C}[[X]](M)$. On note $\mathcal{I}_{M}$ l'idéal engendré par ces éléments sur $\mathbb{C}[[X]](M)$. On suppose que la dimension de l'espace vectoriel $\mathbb{C}[[X]](M) / \mathcal{I}_{M}$, notée $\mu$, est finie et non nulle. 
Soient $e_{1}, \ldots, e_{\mu}$ des monômes tels que leurs classes d'équivalence dans $\mathbb{C}[[X]](M) / \mathcal{I}_{M}$ forment une base de l'espace vectoriel $\mathbb{C}[[X]](M) / \mathcal{I}_{M}$. Alors, pour tout élément $g(X)$ de $\mathbb{C}[[X]](M)$, il existe des séries $R_{i}\left(u_{1}, \ldots, u_{k}\right)$, avec $1 \leq i \leq \mu$, de $\mathbb{C}[[u]]\left(M^{(\mu)}\right)$ telles que l'on ait

$$
g(X)=\sum_{i=1}^{\mu} R_{i}\left(F_{1}, \ldots, F_{k}\right) e_{i}(X) .
$$

Preuve. Soit $\Phi=\left(\Phi_{1}, \ldots, \Phi_{p}\right)$ une bonne base standard de $\mathcal{I}_{M}$. D'après $\left[9\right.$, Corollaire 2], il existe, pour tout $i=1, \ldots, p$, des séries $h_{i, j}, 1 \leq j \leq k$, de $\mathbb{C}[[X]](M)$ telles que l'on ait

$$
\Phi_{i}(X)=\sum_{j=1}^{k} h_{i, j}(X) F_{j}(X) .
$$

Soit $v=\left(v_{1}, \ldots, v_{k}\right)$ des nouvelles variables. On pose alors, pour tout $i=$ $1, \ldots, p$,

$$
\widetilde{F}_{i}\left(X, v^{\mu}\right)=\Phi_{i}(X)-\sum_{j=1}^{k} h_{i, j}(X) v_{j}^{\mu} .
$$

Clairement, par construction on a l'égalité $\operatorname{ord}\left(\widetilde{F}_{i}(X, v)\right)=\operatorname{ord}\left(\Phi_{i}(X)\right)$. Soit $g(X)$ un élément de $\mathbb{C}[[X]](M)$. Le théorème $\mathrm{A}$ de division dans $\mathbb{C}[[X, v]](M)$ et l'algorithme de division formelle de [1] permettent d'écrire l'égalité

$$
g(X)=\sum_{i=1}^{p} q_{i}\left(X, v_{1}^{\mu}, \ldots, v_{k}^{\mu}\right) \widetilde{F}_{i}\left(X, v^{\mu}\right)+\sum_{i=1}^{\mu} H_{i}\left(v_{1}^{\mu}, \ldots, v_{k}^{\mu}\right) m_{i}(X)
$$

avec, pour tout $1 \leq i \leq \mu, H_{i}\left(v_{1}^{\mu}, \ldots, v_{k}^{\mu}\right)$ dans $\mathbb{C}\left[\left[v_{1}, \ldots, v_{k}\right]\right](M)$. Pour vérifier que les séries $q_{i}$ et $h_{i}$ de l'égalité $(2.2)$ sont des séries en les variables $X$ et $v_{1}^{\mu}, \ldots, v_{k}^{\mu}$, il suffit de reprendre l'algorithme de division formelle de [1]. On écrit, pour tout $1 \leq i \leq \mu$,

$$
H_{i}\left(v_{1}^{\mu}, \ldots, v_{k}^{\mu}\right)=\sum_{J \in \mathbb{N}^{k}} h_{J}^{(i)} v_{1}^{\mu j_{1}} \ldots v_{k}^{\mu j_{p}} .
$$

Par hypothèse, on a donc l'existence d'une constante $C_{0}$ telle que l'on ait

$$
\sup _{J \in \mathbb{N}^{k}} \frac{\left|h_{J}^{(i)}\right|}{M_{\mu\left(j_{1}+\ldots+j_{k}\right)}} \leq C_{0} .
$$

On pose $u_{i}=v_{i}^{\mu}$ pour tout $1 \leq i \leq k$. D'après $(2.3)$, les séries $H_{k}\left(u_{1}, \ldots, u_{p}\right)$ appartiennent à $\mathbb{C}\left[\left[u_{1}, \ldots, u_{k}\right]\right]\left(M^{(\mu)}\right)$. De $(2.2)$, on tire l'égalité

$$
\begin{aligned}
g(X)= & \sum_{i=1}^{p} q_{i}\left(X, u_{1}, \ldots, u_{p}\right)\left(\Phi_{i}(X)-\sum_{j=1}^{k} h_{i, j}(X) u_{j}\right) \\
& +\sum_{k=1}^{\mu} H_{k}\left(u_{1}, \ldots, u_{p}\right) m_{k}(X) .
\end{aligned}
$$


En posant $u_{i}=F_{i}(X)$, pour tout $1 \leq i \leq k$, dans (2.4), on obtient

$$
g(X)=\sum_{i=1}^{\mu} H_{i}\left(F_{1}, \ldots, F_{k}\right) m_{i}(X) .
$$

En effectuant un calcul similaire pour les $e_{i}, 1 \leq i \leq \mu$, on a

$$
e_{i}(X)=\sum_{j=1}^{\mu} T_{i, j}\left(F_{1}, \ldots, F_{k}\right) m_{j}(X)
$$

avec $T_{i, j}\left(u_{1}, \ldots, u_{k}\right)$ dans $\mathbb{C}[[u]]\left(M^{(\mu)}\right)$. Soit $T(X)$ la matrice à $\mu$ lignes et $\mu$ colonnes de composantes $T_{i, j}\left(F_{1}, \ldots, F_{k}\right)$. Pour obtenir le théorème, on montre que $T(X)$ est une matrice inversible au sens des séries formelles. On note $D(X)=\operatorname{det}(T(X))$. En reprenant les égalités (2.1) et $(2.2)$ et en y posant $v=0$, on vérifie que l'égalité $D(0)=0$ implique l'existence de scalaires $\lambda_{i}, 1 \leq i \leq \mu$, non tous nuls tels que $\sum_{i=1}^{\mu} \lambda_{i} e_{i}(X) \in \mathcal{I}_{M}$, ce qui est impossible par hypothèse.

3. Remarques. (a) Dans le cas $M=\mathbf{1}$, c'est-à-dire $M_{n}=1$ pour tout entier $n$, le théorème 2 n'est autre que le théorème classsique de préparation de Malgrange [8].

(b) Ce théorème est à rapprocher du théorème 59 de [3]. Dans cet énoncé de [3], le nombre de variables est égal au nombre de fonctions $F_{i}$. Dans le théorème 2, cette hypothèse n'est pas nécessaire.

(c) On se place dans le cas d'une variable $x$. Clairement, toute série formelle $f(x)$ de $\mathbb{C}[[x]](M)$ se décompose de la façon suivante :

$$
f(x)=R_{1}\left(x^{2}\right) \cdot 1+R_{2}\left(x^{2}\right) \cdot x
$$

avec $R_{1}(u)$ et $R_{2}(u)$ dans $\left.\mathbb{C}[u]\right]\left(M^{(2)}\right)$. De plus, dans ce cas, on a l'information supplémentaire suivante : $R_{1}\left(x^{2}\right)$ et $R_{2}\left(x^{2}\right)$ appartiennent aussi à $\mathbb{C}[[x]](M)$. Le théorème 4 généralise en quelque sorte ce phénomène.

4. ThÉorème. Soit $\mathcal{I}_{M}$ un idéal de $\mathbb{C}[[X]](M)$. On suppose que la dimension de l'espace vectoriel $\mathbb{C}[[X]](M) / \mathcal{I}_{M}$, notée $\mu$, est finie et non nulle. Soit $\Phi=\left(\Phi_{1}, \ldots, \Phi_{p}\right)$ une bonne base standard de $\mathcal{I}_{M}$. Soient $e_{1}, \ldots, e_{\mu}$ des monômes tels que leurs classes d'équivalence dans $\mathbb{C}[[X]](M) / \mathcal{I}_{M}$ forment une base de l'espace vectoriel $\mathbb{C}[[X]](M) / \mathcal{I}_{M}$. On note $d=\max _{i=1, \ldots, p}\left(d_{i}\right)$ avec $d_{i}=\operatorname{ord}\left(\Phi_{i}\right)$ pour $i=1, \ldots, p$. Alors, pour tout $g(X) \in \mathbb{C}[[X]](M)$ il existe des séries $R_{i}\left(u_{1}, \ldots, u_{p}\right)$, avec $1 \leq i \leq \mu$, de $\mathbb{C}\left[\left[u_{1}, \ldots, u_{p}\right]\right]\left(M^{(d)}\right)$ telles que l'on ait

$$
g(X)=\sum_{i=1}^{\mu} R_{i}\left(\Phi_{1}, \ldots, \Phi_{p}\right) e_{i}(X) .
$$

De plus, pour tout $1 \leq i \leq \mu$, la série $R_{i}\left(\Phi_{1}(X), \ldots, \Phi_{p}(X)\right)$ appartient à $\mathbb{C}[[X]](M)$. 
Preuve. Pour tout $1 \leq i \leq p$, on pose $F_{i}\left(X, v_{i}\right)=\Phi_{i}(X)-v_{i}^{d_{i}}$. Clairement, par construction, on a l'égalité $\operatorname{ord}\left(F_{i}\left(X, v_{i}\right)\right)=\operatorname{ord}\left(\Phi_{i}(X)\right)$. De la même manière que dans la preuve du théorème 2 , on a l'égalité

$$
\begin{aligned}
g(X)= & \sum_{i=1}^{p} q_{i}\left(X, v_{1}^{d_{1}}, \ldots, v_{p}^{d_{p}}\right)\left(\Phi_{i}(X)-v_{i}^{d_{i}}\right) \\
& +\sum_{k=1}^{\mu} R_{k}\left(v_{1}^{d_{1}}, \ldots, v_{p}^{d_{p}}\right) m_{k}(X)
\end{aligned}
$$

avec, pour tout $1 \leq k \leq \mu, R_{k}\left(v_{1}^{d_{1}}, \ldots, v_{p}^{d_{p}}\right)$ dans $\mathbb{C}\left[\left[v_{1}, \ldots, v_{p}\right]\right](M)$. On écrit alors, pour tout $1 \leq k \leq \mu$,

$$
R_{k}\left(v_{1}^{d_{1}}, \ldots, v_{p}^{d_{p}}\right)=\sum_{J \in \mathbb{N}^{p}} r_{J}^{(k)} v_{1}^{d_{1} j_{1}} \ldots v_{p}^{d_{p} j_{p}} .
$$

Par hypothèse, on a donc l'existence d'une constante $C_{0}$ telle que l'on ait

$$
\sup _{J \in \mathbb{N}^{p}} \frac{\left|r_{J}^{(k)}\right|}{M_{d_{1} j_{1}+\ldots+d_{p} j_{p}}} \leq C_{0}
$$

On pose $u_{i}=v_{i}^{d_{i}}$, pour tout $1 \leq i \leq p$. D'après [12, C, 1.8], pour tout $1 \leq k \leq \mu$, les séries $R_{k}\left(\Phi_{1}(X), \ldots, \Phi_{p}(X)\right)$ appartiennent à $\mathbb{C}[[X]](M)$. Clairement, de (4.2), on déduit aussi que, pour $1 \leq k \leq \mu, R_{k}\left(u_{1}, \ldots, u_{p}\right)$ appartient à $\mathbb{C}\left[\left[u_{1}, \ldots, u_{p}\right]\right]\left(M^{(d)}\right)$. De $(4.1)$, on tire l'égalité

$$
g(X)=\sum_{i=1}^{p} q_{i}\left(X, u_{1}, \ldots, u_{p}\right)\left(\Phi_{i}(X)-u_{i}\right)+\sum_{k=1}^{\mu} R_{k}\left(u_{1}, \ldots, u_{p}\right) m_{k}(X) .
$$

En posant $u_{i}=\Phi_{i}(X)$, pour tout $1 \leq i \leq p$, dans (4.4), on obtient

$$
g(X)=\sum_{k=1}^{\mu} R_{k}\left(\Phi_{1}, \ldots, \Phi_{p}\right) m_{k}(X) .
$$

On termine comme dans la preuve du théorème 2 .

Dans le paragraphe suivant, on montre qu'il est indispensable de bien présenter l'idéal pour obtenir l'information supplémentaire, après recomposition, donnée par le théorème 4 . Pour cela, on donne un exemple d'application $F=\left(F_{1}, F_{2}\right)$ et de série $g$ où, dans une décomposition comme dans le théorème 2 , les séries $R_{k}\left(F_{1}, \ldots, F_{p}\right)$ n'appartiennent pas à $\mathbb{C}[[X]](M)$.

5. Exemple. On pose $M_{n}=n$ !. On se place en deux variables $X$ et $Y$. On pose

$$
F_{1}(X, Y)=X+Y^{3} \quad \text { et } \quad F_{2}(X, Y)=X^{4} .
$$

On note $\mathcal{I}_{M}$ l'idéal engendré par $\left(F_{1}, F_{2}\right)$ sur $\mathbb{C}[[X, Y]]$. On vérifie facilement que la dimension de l'espace vectoriel $\mathbb{C}[[X, Y]](M) / \mathcal{I}_{M}$ est égale à 12 . En utilisant la caractérisation donnée dans [10], on remarque que $\left(F_{1}, F_{2}\right)$ ne 
constitue pas une bonne base standard de l'idéal $\mathcal{I}_{M}$. On vérifie facilement que les monômes $X^{i} Y^{j}$, pour $i \leq 3$ et $j \leq 2$, forment une base de l'espace vectoriel $\mathbb{C}[[X, Y]] / \mathcal{I}_{M}$. On pose

$$
\begin{gathered}
e_{1}=1, \quad e_{2}=X, \quad e_{3}=Y, \quad e_{4}=X^{2}, \quad e_{5}=X Y, \quad e_{6}=Y^{2}, \quad e_{7}=X^{3}, \\
e_{8}=X^{2} Y, \quad e_{9}=X Y^{2}, \quad e_{10}=X^{3} Y, \quad e_{11}=X^{2} Y^{2}, \quad e_{12}=X^{3} Y^{2} .
\end{gathered}
$$

On va donc montrer que l'on ne peut pas espérer, sous les hypothèses du théorème 2 , les conclusions du théorème 4 .

Soit $g(X, Y)=\sum_{n=0}^{\infty} M_{n} Y^{n}$. On va donner une représentation de $g$ de la forme

$$
g(X, Y)=\sum_{i=1}^{12} R_{i}\left(F_{1}, F_{2}\right) e_{i}(X, Y)
$$

où $R_{1}\left(F_{1}, F_{2}\right)$ n'appartient pas à $\mathbb{C}[[X, Y]](M)$. De plus, au sens des séries formelles, cette représentation est unique car le nombre de séries est égal au nombre de variables [13]. Pour cela, comme le suggère la preuve du théorème 4 , on effectue la division formelle de $g(X, Y)$ par $X+Y^{3}-u_{1}$ et $X^{4}-u_{2}$. On a déjà facilement l'égalité, après division par $X+Y^{3}-u_{1}$,

$$
\begin{aligned}
g(X, Y)= & \left(X+Y^{3}-u_{1}\right)\left(\sum_{k=1}^{\infty} \sum_{n=0}^{\infty}(-1)^{k+1} M_{n+3 k} Y^{n}\left(X-u_{1}\right)^{k-1}\right) \\
& +\sum_{k=0}^{\infty}(-1)^{k}\left(X-u_{1}\right)^{k}\left(M_{3 k}+M_{3 k+1} Y+M_{3 k+2} Y^{2}\right) .
\end{aligned}
$$

Pour achever la division, il reste à diviser le terme

$$
\sum_{k=0}^{\infty}(-1)^{k}\left(X-u_{1}\right)^{k}\left(M_{3 k}+M_{3 k+1} Y+M_{3 k+2} Y^{2}\right)
$$

par $X^{4}-u_{2}$. On ne s'intéresse qu'au terme $R_{1}\left(u_{1}, u_{2}\right)$. Il suffit donc de considérer la division de $\sum_{k=0}^{\infty}(-1)^{k}\left(X-u_{1}\right)^{k} M_{3 k}$ par $X^{4}-u_{2}$. Or, on a

$$
\sum_{k=0}^{\infty}(-1)^{k}\left(X-u_{1}\right)^{k} M_{3 k}=\sum_{k=0}^{\infty} \sum_{i=0}^{k}\left(\begin{array}{l}
k \\
i
\end{array}\right)(-1)^{i} X^{i} u_{1}^{k-i} M_{3 k} .
$$

La division par $X^{4}-u_{2}$ revient à remplacer $X^{4}$ par $u_{2}$. Clairement on obtiendra alors le terme $R_{1}\left(u_{1}, u_{2}\right)$ de la division par $X^{4}-u_{2}$ du terme

$$
\sum_{k=0}^{\infty} \sum_{\substack{i=0 \\
i=4 i^{\prime}}}^{k}\left(\begin{array}{c}
k \\
i
\end{array}\right) X^{4 i^{\prime}} u_{1}^{k-i} M_{3 k}
$$


On obtient alors

$$
R_{1}\left(u_{1}, u_{2}\right)=\sum_{k=0}^{\infty} \sum_{\substack{i=0 \\
i=4 i^{\prime}}}^{k}\left(\begin{array}{c}
k \\
i
\end{array}\right) u_{2}^{i^{\prime}} u_{1}^{k-i} M_{3 k}
$$

On a donc

$$
R_{1}\left(X+Y^{3}, X^{4}\right)=\sum_{k=0}^{\infty} \sum_{\substack{i=0 \\
i=4 i^{\prime}}}^{k}\left(\begin{array}{c}
k \\
i
\end{array}\right) X^{4 i^{\prime}}\left(X+Y^{3}\right)^{k-i} M_{3 k} .
$$

On en tire

$$
R_{1}\left(X+Y^{3}, X^{4}\right)=\sum_{k=0}^{\infty} \sum_{\substack{i=0 \\
i=4 i^{\prime}}}^{k}\left(\begin{array}{c}
k \\
i
\end{array}\right) X^{4 i^{\prime}} X^{k-4 i^{\prime}} M_{3 k}+T(X, Y)
$$

avec la série $T(X, Y)$ ne comportant pas de termes en $X^{n}, n \in \mathbb{N}$, dans son développement. Or, on a

$$
\sum_{k=0}^{\infty} \sum_{\substack{i=0 \\
i=4 i^{\prime}}}^{k}\left(\begin{array}{l}
k \\
i
\end{array}\right) X^{4 i^{\prime}} X^{k-4 i^{\prime}} M_{3 k}=\sum_{k=0}^{\infty} M_{3 k} X^{k}\left(\sum_{\substack{i=0 \\
i=4 i^{\prime}}}^{k}\left(\begin{array}{l}
k \\
i
\end{array}\right)\right) .
$$

On en déduit que $R_{1}\left(X+Y^{3}, X^{4}\right)$ n'appartient pas à $\mathbb{C}[[X, Y]](M)$.

\section{Application à l'étude de certaines relations formelles}

6. Quelques rappels d'algèbre commutative. Soit $A$ un anneau local noethérien d'idéal maximal $\underline{m}$. Une chaîne d'idéaux premiers dans $A$ est une suite finie croissante

$$
\mathcal{P}_{0} \subset \mathcal{P}_{1} \subset \ldots \subset \mathcal{P}_{r}
$$

d'idéaux premiers tels que $\mathcal{P}_{i} \neq \mathcal{P}_{i+1}$ pour $0 \leq i \leq r-1$. On appelle dimension de $A$, et l'on note $\operatorname{dim}(A)$, la borne supérieure des longueurs des chaînes d'idéaux premiers dans $A$. On a donc, par exemple, $\operatorname{dim}(\mathbb{C}[[X]])=$ $\operatorname{dim}(\mathbb{C}[[X]](M))=s($ cf. $[15])$.

Un idéal $I$ de $A$ est un idéal de définition si $I$ est inclus dans $\underline{m}$ et $I$ contient une puissance de $\underline{m}$.

On considère $\operatorname{Der}(A)$, le $A$-module des dérivations de l'anneau $A$ dans $A$. Pour tout idéal $I$ de $A$, on pose $\operatorname{Der}(I)=\{D \in \operatorname{Der}(A): D(I) \subset I\}$. Clairement si on a $A=\mathbb{C}[[X]]$ ou $A=\mathbb{C}[[X]](M), \operatorname{Der}(A)$ est engendré par les dérivations partielles $\partial / \partial X_{i}$ pour $1 \leq i \leq s$.

On établit d'abord le théorème suivant qui généralise un résultat de $\mathrm{A}$. Płoski [14].

7. ThÉorème. Soit $\mathcal{I}_{M}$ un idéal premier de $\mathbb{C}[[X]](M)$. Soient $f_{1}$ et $f_{2}$ des éléments de $\underline{m}_{X, M}$. On suppose que, pour toutes dérivations $D, D^{\prime}$ de 
$\operatorname{Der}\left(\mathcal{I}_{M}\right)$, la série $D f D^{\prime} g-D^{\prime} g D f$ appartient à $\mathcal{I}_{M}$. Alors il existe un entier $d$ et une série $P(u, v) \neq 0$ de $\mathbb{C}[[u, v]]\left(M^{(d)}\right)$ telle que $P\left(f_{1}, f_{2}\right)$ appartient $\grave{a} \mathcal{I}_{M}$.

La preuve de ce théorème repose en partie sur la proposition suivante.

8. Proposition. Soit $\mathcal{I}_{M}$ un idéal premier de $\mathbb{C}[[X]](M)$, notons $k=$ $\operatorname{dim}\left(\mathbb{C}[[X]](M) / \mathcal{I}_{M}\right)$ et soit $h=\left(h_{1}, \ldots, h_{k}\right)$ un système de paramètres modulo $\mathcal{I}_{M}$ (c'est-à-dire l'idéal $\left(h_{1}, \ldots, h_{k}\right) \mathbb{C}[[X]](M)+\mathcal{I}_{M}$ est un idéal de définition dans $\mathbb{C}[[X]](M))$. Alors il existe des dérivations $D_{1}, \ldots, D_{k}$ de $\operatorname{Der}\left(\mathcal{I}_{M}\right)$ telles que, pour tous $i, j, 1 \leq i, j \leq k, i \neq j$, on ait $D_{i} h_{j} \in \mathcal{I}_{M}$ et $D_{i} h_{i} \notin \mathcal{I}_{M}$.

Preuve. On adapte celle faite par A. Płoski dans le cas analytique [14] en reprenant des idées déjà utilisées par l'auteur dans [9] et [12].

9. Preuve du théorème $\%$. Comme $\mathbb{C}[[X]](M)$ est un anneau noethérien [2], il existe des séries $\phi=\left(\phi_{1}, \ldots, \phi_{p}\right)$ qui engendrent l'idéal $\mathcal{I}_{M}$. Soit $h=\left(h_{1}, \ldots, h_{k}\right)$ un système de paramètres modulo $\mathcal{I}_{M}$. On pose $Y=$ $\left(Y_{1}, \ldots, Y_{k}\right)$. Soit $f \in \underline{m}_{X, M}$. Par hypothèse, on a donc

$$
\operatorname{dim}_{\mathbb{C}}\left(\mathbb{C}[[X]](M) /\left(h_{1}, \ldots, h_{k}\right) \mathbb{C}[[X]](M)+\mathcal{I}_{M}\right)=\mu<\infty .
$$

Soit alors $e_{1}, \ldots, e_{\mu}$ une base correspondante. On applique le théorème de préparation 2. Pour tout $j=1, \ldots, \mu$, il existe des séries $R_{i, j}\left(Y, t_{1}, \ldots, t_{p}\right)$ de $\mathbb{C}[[Y, t]]\left(M^{(\mu)}\right)$ telles que l'on ait

$$
f \cdot e_{j}=\sum_{i=1}^{\mu} R_{i, j}(h, \phi) e_{i} .
$$

Ainsi on a, pour tout $j=1, \ldots, \mu$,

$$
\sum_{i=1}^{\mu}\left(R_{i, j}(h, \phi)-f \cdot \delta_{i, j}\right) \cdot e_{j}=0 \quad\left(\delta_{i, j} \text { est le symbôle de Kronecker }\right) .
$$

Par la règle de Cramer, il existe un polynôme $D(Y, t, v)=\operatorname{det}\left(R_{i, j}(Y, t)-\right.$ $\left.v \delta_{i, j}\right)$ de $\mathbb{C}[[Y, t]]\left(M^{(\mu)}\right)[v]$ tel que $D(h, \phi, f)=0$. On obtient alors, puisque $\phi$ engendre $\mathcal{I}_{M}$, un polynôme $\bar{D}(Y, v)=v^{\mu}+d_{1}(Y) v^{\mu-1}+\ldots+d_{\mu}(Y)$ de $\mathbb{C}[[Y]]\left(M^{(\mu)}\right)[v]$ vérifiant $\bar{D}(h, f) \in \mathcal{I}_{M}$. On en déduit aussi $d_{\mu}(0)=0$. On peut donc supposer, quitte à appliquer le théorème de préparation de Weierstrass dans $\mathbb{C}[[Y]]\left(M^{(\mu)}\right)[v]$ (cf. $\left.[10,3.3]\right)$ que $\bar{D}(Y, v)$ est distingué. On pose

$$
J_{M}=\left\{S(Y, v) \in \mathbb{C}[[Y]]\left(M^{(\mu)}\right)[v]: S(h, f) \in \mathcal{I}_{M}\right\} .
$$

On a $\bar{D}(Y, v) \in J_{M}$ et $J_{M}$ est un idéal premier. On choisit $P_{f}(Y, v)$, de degré minimal $l>0$, appartenant à $J_{M}$. Comme précédemment, on peut supposer $P_{f}(Y, v)$ distingué. Par minimalité de $l$ et toujours grâce à [10, 3.3], on a 
aussi, pour toute série $S(Y, v)$ de $J_{M}$,

$$
S(Y, v)=Q(Y, v) P_{f}(Y, v)
$$

avec $Q(Y, v)$ dans $\mathbb{C}[[Y, v]]\left(M^{(\mu)}\right)$. Soient alors les dérivations $D_{i}$, pour $i=$ $1, \ldots, k$, données par la proposition 8 appliquée à $\mathcal{I}_{M}$. On montre d'abord que, pour tout $i=1, \ldots, k$, la condition $D_{i} f \in \mathcal{I}_{M}$ entraîne $\partial P_{f} / \partial Y_{i}=0$. Sinon, comme $P_{f}(h, f)$ appartient à $\mathcal{I}_{M}$, on a $D_{i}\left(P_{f}(h, f)\right) \in \mathcal{I}_{M}$. On en déduit

$$
\frac{\partial P_{f}}{\partial Y_{i}}(h, f) D_{i} h_{i}+\sum_{\substack{j=1 \\ j \neq i}}^{k} \frac{\partial P_{f}}{\partial Y_{j}}(h, f) D_{i} h_{j}+\frac{\partial P_{f}}{\partial v}(h, f) D_{i} f \in \mathcal{I}_{M} .
$$

Comme $\mathcal{I}_{M}$ est premier, des relations $D_{i} h_{i} \notin \mathcal{I}_{M}, D_{i} h_{j} \in \mathcal{I}_{M}$ et de l'hypothèse $D_{i} f \in \mathcal{I}_{M}$, on tire

$$
\frac{\partial P_{f}}{\partial Y_{i}}(h, f) \in \mathcal{I}_{M}
$$

Cela implique, d'après (9.3) et (9.4), l'existence d'une série $Q(Y, v)$ dans $\mathbb{C}[[Y, v]]\left(M^{(\mu)}\right)$ telle que l'on ait

$$
\frac{\partial P_{f}}{\partial Y_{i}}(Y, v)=Q(Y, v) P_{f}(Y, v) .
$$

Or le degré en $v$ de $\partial P_{f} / \partial Y_{i}$ est strictement inférieur à $l$, car $P_{f}$ est distingué. On a donc une contradiction et $\partial P_{f} / \partial Y_{i}=0$. Ainsi $D_{i} f \in \mathcal{I}_{M}$ entraîne $\partial P_{f} / \partial Y_{i}=0$

Soient alors $f_{1}$ et $f_{2}$ dans $\underline{m}_{X, M}$. On suppose donc $D f_{1} D^{\prime} f_{2}-D^{\prime} f_{1} D f_{2} \in$ $\mathcal{I}_{M}$ pour toutes dérivations $D, D^{\prime}$ de $\operatorname{Der}\left(\mathcal{I}_{M}\right)$. On peut supposer $f_{1} \notin \mathcal{I}_{M}$. Il existe un système de paramètres $h_{1}, \ldots, h_{k}$ avec $h_{1}=f_{1}$. On a donc $D_{1} f_{1} \notin \mathcal{I}_{M}$ et $D_{i} f_{1} \in \mathcal{I}_{M}$ pour $i=2, \ldots, k$. Les relations $D_{i} f_{2} D_{1} f_{1}-$ $D_{i} f_{1} D_{1} f_{2} \in \mathcal{I}_{M}$ impliquent $D_{i} f_{2} \in \mathcal{I}_{M}$ pour $i=2, \ldots, k$. D'après ce qui précède, on a alors $\partial P_{f_{2}} / \partial Y_{i}=0,2 \leq i \leq k$. La série $P_{f_{2}}$ ne dépend donc que des variables $Y_{1}$ et $v$. Ceci achève la preuve du théorème 7 .

10. Remarque. Dans le cas où $M=1$, on a aussi $M^{(d)}=1$ et le théorème 7 n'est autre que le théorème de A. Płoski [14]. Pour une suite $M$ quelconque, on observe une perte de régularité, mesurée par l'entier $d$, sur la classe $M$. Dans le cas où l'idéal $\mathcal{I}_{M}$ du théorème est réduit à $\{0\}$, on peut donner une estimation précise de cette perte.

11. ThÉORÈme. Soient $f_{1}$ et $f_{2}$ des éléments de $\underline{m}_{X, M}$. On suppose $\operatorname{ord}\left(f_{1}\right) \leq \operatorname{ord}\left(f_{2}\right)$ et $\operatorname{rang}\left(\partial f_{i} / \partial X_{j}\right)_{1 \leq i \leq 2,1 \leq j \leq s}=1$ (ceci signifie qu'au sens des séries formelles tous les mineurs d'ordre 2 sont nuls). Alors il existe une série $P(u, v) \neq 0$ de $\mathbb{C}[[u, v]]\left(M^{\left(\operatorname{ord}\left(f_{1}\right)\right)}\right)$ telle que $P\left(f_{1}, f_{2}\right)=0$.

Preuve. L'hypothèse de rang signifie exactement $D f_{1} D^{\prime} f_{2}-D^{\prime} f_{2} D f_{1}=0$ pour toutes les dérivations $D, D^{\prime}$. Le théorème 11 correspond donc au 
théorème 7 avec $\mathcal{I}_{M}=(0)$. Il suffit simplement de prouver $d=\operatorname{ord}\left(f_{1}\right)$. On reprend donc la démonstration du théorème 7 . On peut supposer $h_{1}=f_{1}$. On peut clairement choisir pour $h_{2}, \ldots, h_{k}$ des séries d'ordre 1 . On a alors $\mu=\operatorname{dim}_{\mathbb{C}}\left(\mathbb{C}[[X]](M) /\left(h_{1}, \ldots, h_{k}\right) \mathbb{C}[[X]](M)\right)=\operatorname{ord}\left(f_{1}\right) ;$ soient $e_{1}, \ldots, e_{\mu}$ des monômes formant une base correspondante. Clairement on a

$$
\operatorname{ord}\left(e_{i}\right) \leq \mu-1 \quad \text { pour tout } i=1, \ldots, \mu .
$$

On construit alors des séries $R_{i, j}(Y), 1 \leq i, j \leq \mu$, comme dans (9.1). D'après le théorème 2 , pour tout $j=1, \ldots, \mu$, il existe des séries $R_{i, j}(Y)$ de $\mathbb{C}[[Y]]\left(M^{(\mu)}\right)$ telles que l'on ait

$$
f_{2} \cdot e_{j}=\sum_{i=1}^{\mu} R_{i, j}(h) e_{i} .
$$

Cette égalité s'obtient, d'après (2.4), en écrivant

$$
f_{2}(X) \cdot e_{j}=\sum_{i=1}^{k}\left(u_{i}-h_{i}(X)\right) Q_{i, j}(u, X)+\sum_{i=1}^{\mu} R_{i, j}(u) e_{i} .
$$

En posant $u=0$ dans cette dernière égalité, on obtient

$$
f_{2}(X) \cdot e_{j}=-\sum_{i=1}^{k} h_{i}(X) Q_{i, j}(u, X)+\sum_{i=1}^{\mu} R_{i, j}(0) e_{i} .
$$

Par des considérations de support dans la division d'Hironaka, on a, pour tout $j=1, \ldots, \mu$,

$$
\operatorname{ord}\left(f_{2} e_{j}\right) \leq \min \left(\operatorname{ord}\left(R_{i, j}(0) e_{i}\right) .\right.
$$

Or, par hypothèse, $\operatorname{ord}\left(f_{2}\right) \geq \mu$. Par conséquent, aussi ord $\left(f_{2} e_{j}\right) \geq \mu$. S'il existe $i$ tel que $R_{i, j}(0) \neq 0$, l'inégalité (11.1) donne une contradiction. Par construction la série $D(Y, v)=\operatorname{det}\left(R_{i, j}(Y)-v \delta_{i, j}\right)$ de $\mathbb{C}[[Y]]\left(M^{(\mu)}\right)[v]$ est donc d'ordre exactement $\mu$. Donc, d'après le théorème $\mathrm{A}$, puisqu'ici $(0, \ldots, 0, \mu)$ est le bon exposant privilégié de $D(Y, v)$, pour toute série $S(Y, v)$ de $\mathbb{C}[[Y, v]]\left(M^{(\mu)}\right)$ on a

$$
S(Y, v)=Q(Y, v) D(Y, v)+R(Y, v)
$$

avec $Q(Y, v)$ et $R(Y, v)$ dans $\mathbb{C}[[Y, v]]\left(M^{(\mu)}\right)$ et $R(Y, v)$ polynômial en $v$ de degré strictement inférieur à $\mu$. On considère alors

$$
J_{M}=\left\{S(Y, v) \in \mathbb{C}[[Y, v]]\left(M^{(\mu)}\right): S\left(h, f_{2}\right)=0\right\} .
$$

On peut choisir $R_{f_{2}}(Y, v)$ de $J_{M}$ dans $\mathbb{C}[[Y]]\left(M^{(\mu)}\right)[v]$ distingué et de degré minimal. On peut aussi choisir $P_{f_{2}}(Y, v)$ de $J_{M}$ dans $\mathbb{C}[[Y]]\left(M^{(\mu)}\right)[v]$ distingué et de degré minimal parmi ceux qui vérifient

$$
\operatorname{ord}\left(P_{f_{2}}\right)=\operatorname{deg}_{v}\left(P_{f_{2}}\right) .
$$


Clairement on a

$$
\operatorname{deg}_{v}\left(R_{f_{2}}\right) \leq \operatorname{deg}_{v}\left(P_{f_{2}}\right) .
$$

De plus, par le théorème [10,3.3], on a

$$
P_{f_{2}}(Y, v)=Q_{1}(Y, v) R_{f_{2}}(Y, v)+R_{1}(Y, v)
$$

avec $Q_{1}(Y, v)$ et $R_{1}(Y, v)$ dans $\mathbb{C}[[Y, v]]\left(M^{(\mu)}\right)$ et $R_{1}(Y, v)$ polynômial en la variable $v$ de degré strictement inférieur à $\operatorname{deg}_{v}\left(R_{f_{2}}\right)$. De (11.2), on tire $R_{1}\left(h, f_{2}\right)=0$. Ainsi par minimalité du degré de $R_{f_{2}}$, on a $R_{1}(Y, v)=0$. Grâce à des considérations d'ordre, l'égalité (11.2) permet donc d'écrire

$$
\operatorname{deg}_{v}\left(P_{f_{2}}\right)=\operatorname{deg}_{v}\left(R_{f_{2}}\right) \text {. }
$$

Pour toute série $S(Y, v)$ de $\mathbb{C}[[Y, v]]\left(M^{(\mu)}\right)$, on peut alors appliquer le théorème $\mathrm{A}$ et écrire

$$
S(Y, v)=Q(Y, v) P_{f_{2}}(Y, v)+R(Y, v)
$$

avec $Q(Y, v)$ et $R(Y, v)$ dans $\mathbb{C}[[Y, v]]\left(M^{(\mu)}\right)$ et $R(Y, v)$ polynômial en la variable $v$ de degré strictement inférieur à $\operatorname{deg}_{v}\left(P_{f_{2}}\right)$. On en déduit, par minimalité du degré, $R(Y, v)=0$ et donc que $J_{M}$ est engendré par $P_{f_{2}}$. On montre alors, comme dans la preuve du théorème 7 , que $P_{f_{2}}$ ne dépend que des variables $Y_{1}$ et $v$.

12. Exemple. On pose $f_{1}(x)=\sum_{n=1}^{\infty} M_{2 n} x^{2 n}$ et $f_{2}(x)=x^{2}$. On a $f_{1}$ et $f_{2}$ dans $\mathbb{C}[[x]](M), \operatorname{rang}\left(\partial f_{i} / \partial x\right)_{1 \leq i \leq 2}=1$ et $\min \left(\operatorname{ord}\left(f_{1}\right), \operatorname{ord}\left(f_{2}\right)\right)=2$. Soit $P(u, v)=\sum_{n=1}^{\infty} M_{2 n} v^{n}-u$. On vérifie sans difficulté $P \in \mathbb{C}[[u, v]]\left(M^{(2)}\right)$ et $P\left(f_{1}, f_{2}\right)=0$.

On obtient facilement d'autres propriétés de l'application $F=\left(f_{1}, f_{2}\right)$. On pose

$$
\mathcal{R}=\left\{S(u, v) \in \mathbb{C}[[u, v]]: S\left(f_{1}, f_{2}\right)=0\right\} .
$$

Clairement $\mathcal{R}$ est un idéal premier de $\mathbb{C}[[u, v]]$. En fait, $\mathcal{R}$ est l'idéal engendré par $P(u, v)$ sur $\mathbb{C}[[u, v]]$. En effet, si $S(u, v)$ est une série telle que $S\left(f_{1}, f_{2}\right)$ $=0$, on peut écrire (division de Weierstrass par rapport à $u$ )

$$
S(u, v)=Q(u, v)\left(\sum_{n=1}^{\infty} M_{2 n} v^{n}-u\right)+R(v) .
$$

Ainsi l'égalité $S\left(f_{1}, f_{2}\right)=0$ implique $R\left(x^{2}\right)=0$, soit $R(v)=0$, ce qui donne le résultat.

Soit $N$ une suite vérifiant $\left(\mathrm{H}_{1}\right)$ et $\left(\mathrm{H}_{2}\right)$ telle que l'anneau $\mathbb{C}[[X]](N)$ soit strictement inclus dans $\mathbb{C}[[X]]\left(M^{(2)}\right)$. On montre aussi qu'il n'existe pas de série $S(u, v) \neq 0$ dans $\mathbb{C}[[u, v]](N)$ telle que $S\left(f_{1}, f_{2}\right)=0$. Sinon, puisque l'anneau $\mathbb{C}[[u, v]](N)$ est factoriel [9], on peut clairement supposer $S(u, v)$ irréductible dans $\mathbb{C}[[u, v]]$. D'après ce qui précède, il existe $Q(u, v)$ dans $\mathbb{C}[[u, v]]$ telle que $S(u, v)=Q(u, v) P(u, v)$. Comme $S(u, v)$ est irréductible, la série $Q(u, v)$ est inversible et $S(u, v)$ est régulière d'ordre 1 en $u$. Le 
théorème de préparation de Weierstrass dans $\mathbb{C}[[v]](N)[u]$ donne l'existence d'uniques séries $T(u, v)$ inversibles dans $\mathbb{C}[[u, v]](N)$ et $W(v)$ dans $\mathbb{C}[[v]](N)$ telles que l'on ait

$$
S(u, v)=T(u, v)(u-W(v)) .
$$

Par unicité de la représentation, on conclut $W(v)=\sum_{n=1}^{\infty} M_{2 n} v^{n}$, ce qui n'est pas possible car $W(v)$ appartient à $\mathbb{C}[[v]](N)$ a priori. Le polynôme $P(u, v)$ est donc la "meilleure" relation formelle entre $f_{1}$ et $f_{2}$ (au sens de l'échelle de régularité définie par les suites $M$ ).

13. Notations. Dans toute la suite, on considère $F(X)=\left(f_{1}(X), \ldots\right.$, $\left.f_{p}(X)\right)$ une application formelle de $\mathbb{C}^{s}$ dans $\mathbb{C}^{p}$ avec, pour tout $1 \leq i \leq p$, $f_{i}(X)$ dans $\mathbb{C}[[X]](M)$ et $f_{i}(0)=0$. On note $\mathcal{J}_{F}$ la matrice jacobienne de l'application $F$. Soit $\mathcal{R}$ l'idéal des relations entre les $f_{i}, 1 \leq i \leq p$, défini par

$$
\mathcal{R}=\left\{R \in \mathbb{C}\left[\left[u_{1}, \ldots, u_{p}\right]\right]: R \circ F=0\right\} .
$$

On sait bien [5], [6] que si $p=s$ et $\operatorname{rang}\left(\mathcal{J}_{F}\right)=s$, alors $\mathcal{R}$ est réduit à $\{0\}$. En revanche, d'après le théorème $11, \operatorname{si} \operatorname{rang}\left(\mathcal{J}_{F}\right)=1$, alors $\mathcal{R}$ n'est pas réduit à $\{0\}$. De plus, si $M=\mathbf{1}$, c'est-à-dire si l'application $F$ est analytique, A. M. Gabrielov a montré que l'idéal $\mathcal{R}$ était engendré par des séries convergentes [6]. Comme dans l'exemple 12, on généralise ce résultat pour une suite $M$ quelconque.

14. Proposition. Soit $M$ une suite de réels positifs vérifiant $\left(\mathrm{H}_{1}\right)$ et $\left(\mathrm{H}_{2}\right)$. Soit une application $F(X)=\left(f_{1}(X), \ldots, f_{p}(X)\right)$ de $\mathbb{C}^{s}$ dans $\mathbb{C}^{p}$ avec, pour tout $1 \leq i \leq p, f_{i}(X)$ dans $\mathbb{C}[[X]](M)$. On note $\mathcal{J}_{F}$ sa matrice jacobienne. On suppose $\operatorname{rang}\left(\mathcal{J}_{F}\right)=1$. Alors l'idéal $\mathcal{R}$ des relations entre les $f_{i}$,

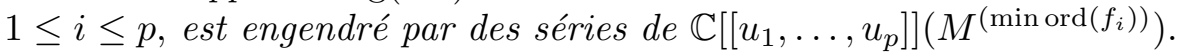

Preuve. C'est une conséquence directe du théorème 11. Sans restreindre la généralité, on suppose $\min \left(\operatorname{ord}\left(f_{i}\right)\right)=\operatorname{ord}\left(f_{1}\right)$. D'après la preuve $\mathrm{du}$ théorème 11, il existe $p-1$ polynômes non nuls $P_{i}\left(u_{1}, u_{i}\right)$, pour $i=$ $2, \ldots, p$, de $\mathbb{C}\left[\left[u_{1}\right]\right]\left(M^{\left(\operatorname{ord}\left(f_{1}\right)\right)}\right)\left[u_{i}\right]$ distingués, de degré minimal tels que l'on ait $P_{i}\left(f_{1}, f_{i}\right)=0$ et ord $\left(P_{i}\right)=\operatorname{deg}_{u_{i}}\left(P_{i}\right)$. Par des considérations élémentaires de hauteur, on vérifie que ces séries engendrent l'idéal des relations entre les $f_{i}$.

REMARque. L'exemple 12 montre que la perte de régularité sur la croissance des coefficients est optimale.

15. Notations. On reprend les notations du paragraphe 13. On sait que, si on suppose $p \leq s$ et $\operatorname{rang}\left(\mathcal{J}_{F}\right)=p$, la propriété suivante est vérifiée [11] :

(*) $\quad$ il existe un entier $d$ tel que, pour tout $\mathcal{A}$ de $\mathbb{C}[[X]]$, si $\mathcal{A} \circ F$ appartient à $\mathbb{C}[[X]](M)$ alors $\mathcal{A}$ appartient à $\mathbb{C}[[X]]\left(M^{(d)}\right)$. 
Dans le cas où la matrice jacobienne de l'application $F$ n'est pas de rang maximal, la conclusion précédente est fausse [11]. Dans [11], on pose alors la question suivante : si l'idéal $\mathcal{R}$ des relations entre les $f_{i}, 1 \leq i \leq p$, n'est pas réduit à $\{0\}$, sous quelle hypothèse la propriété suivante est-elle vraie :

(**) il existe un entier $d$ tel que, pour tout $\mathcal{A}$ de $\mathbb{C}[[X]]$, si $\mathcal{A} \circ F$ appartient à $\mathbb{C}[[X]](M)$ alors il existe une série $\widetilde{\mathcal{A}}$ de $\mathbb{C}[[X]]\left(M^{(d)}\right)$ telle que $\widetilde{\mathcal{A}} \circ F=\mathcal{A} \circ F$ ?

On s'intéresse au cas $\operatorname{rang}\left(\mathcal{J}_{F}\right)=1$. On a alors le résultat suivant.

16. ThÉonÈme. Soit $M$ une suite de réels positifs vérifiant $\left(\mathrm{H}_{1}\right)$ et $\left(\mathrm{H}_{2}\right)$. Soit une application $F(X)=\left(f_{1}(X), \ldots, f_{p}(X)\right)$ de $\mathbb{C}^{s}$ dans $\mathbb{C}^{p}$ avec, pour tout $1 \leq i \leq p, f_{i}(X)$ dans $\mathbb{C}[[X]](M)$. On note $\mathcal{J}_{F}$ sa matrice jacobienne. On suppose $\operatorname{rang}\left(\mathcal{J}_{F}\right)=1$. Alors on a $(* *)$ avec $d=\min \left(\operatorname{ord}\left(f_{i}\right)\right)$.

Pour démontrer ce théorème, on utilise le lemme suivant.

17. Lemme. Soit $M$ une suite de réels positifs vérifiant $\left(\mathrm{H}_{1}\right)$ et $\left(\mathrm{H}_{2}\right)$. Soit une application $F(X)=\left(f_{1}(X), \ldots, f_{p}(X)\right)$ de $\mathbb{C}^{s}$ dans $\mathbb{C}^{p}$ avec, pour tout $1 \leq i \leq p, f_{i}(X)$ dans $\mathbb{C}[[X]](M)$. On note $\mathcal{J}_{F}$ sa matrice jacobienne. On suppose aussi $\operatorname{rang}\left(\mathcal{J}_{F}\right)=1$. Alors il existe une application formelle $H(X)=\left(h_{1}(X), \ldots, h_{s}(X)\right)$, de $\mathbb{C}^{s}$ dans $\mathbb{C}^{s}$, à composantes dans $\mathbb{C}[[X]](M)$ et de jacobien non nul telle que $F \circ H$ ne dépende que d'une variable. De plus, on a $\min \left(\operatorname{ord}\left(f_{i} \circ H\right)\right)=\min \left(\operatorname{ord}\left(f_{i}\right)\right)$.

Preuve. C'est un cas particulier du lemme 8 de [11] qui généralise le cas analytique [5].

18. Preuve du théorème 16. Soit $\mathcal{A}$ de $\mathbb{C}[[X]]$ tel que $\mathcal{A} \circ F \in \mathbb{C}[[X]](M)$. Le lemme 17 donne une application $H(X)$, de $\mathbb{C}^{s}$ dans $\mathbb{C}^{s}$, à composantes dans $\mathbb{C}[[X]](M)$ et de jacobien non nul telle que $G=F \circ H$ ne dépende que d'une variable. On note $G=\left(g_{1}, \ldots, g_{p}\right)$. On a $\min \left(\operatorname{ord}\left(g_{i}\right)\right)=\min \left(\operatorname{ord}\left(f_{i}\right)\right)$ $=\mu$. Les séries $g_{i}$, pour $1 \leq i \leq p$, ne dépendent donc que d'une seule variable. Par commodité, on note $x$ cette variable. On note aussi $c=\mathcal{A} \circ G$. Clairement on a

$$
\operatorname{dim}_{\mathbb{C}}\left(\mathbb{C}[[x]](M) /\left(g_{1}(x), \ldots, g_{p}(x)\right) \mathbb{C}[[x]](M)\right)=\mu .
$$

Comme dans (9.2), on en déduit une relation du type

$$
c(x)^{\mu}+r_{1}\left(g_{1}(x), \ldots, g_{p}(x)\right) c(x)^{\mu-1}+\ldots+r_{\mu}\left(g_{1}(x), \ldots, g_{p}(x)\right)=0
$$

avec $r_{i}(u)$ dans $\mathbb{C}[[u]]\left(M^{(\mu)}\right)$ pour tout $i=1, \ldots, \mu$. On pose

$$
\mathcal{R}=\left\{S(u) \in \mathbb{C}[[u]]: S\left(g_{1}, \ldots, g_{p}\right)=0\right\} .
$$

D'après (18.2), la série $\mathcal{A}(u)^{\mu}+r_{1}(u) \mathcal{A}(u)^{\mu-1}+\ldots+r_{\mu}(u)$ appartient à $\mathcal{R}$.

On pose 


$$
\begin{array}{r}
E=\left\{S(u, w)=w^{j}+b_{1}(u) w^{j-1}+\ldots+b_{j}(u) \in \mathbb{C}[[u]]\left(M^{(\mu)}\right)[w]:\right. \\
j \geq 1 \text { et } S(u, \mathcal{A}(u)) \in \mathcal{R}\} .
\end{array}
$$

D'après ce qui précède, l'ensemble $E$ est non vide. Soit alors $P_{\min }(u, w)$ de $E$ de degré minimal $l \geq 1$ en $w$. On écrit

$$
P_{\min }(u, w)=w^{l}+p_{1}(u) w^{l-1}+\ldots+p_{l}(u)
$$

avec $l \geq 1, p_{i}(u) \in \mathbb{C}[[u]]\left(M^{(\mu)}\right)$ pour $i=1, \ldots, l$, et $p_{l}(u) \notin \mathcal{R}$. D'après la proposition 14 , il existe des séries $a_{1}(u), \ldots, a_{m}(u)$ de $\mathbb{C}[[u]]\left(M^{(\mu)}\right)$ qui engendrent l'idéal $\mathcal{R}$. On a donc

$$
\mathcal{A}(u)^{l}+p_{1}(u) \mathcal{A}(u)^{l-1}+\ldots+p_{l-1}(u) \mathcal{A}(u)+p_{l}(u)=\sum_{i=1}^{m} \bar{Q}_{i}(u) a_{i}(u),
$$

avec $\bar{Q}_{i}(u)$ dans $\mathbb{C}[[u]]$ pour $i=1, \ldots, m$. D'après le théorème d'approximation d'Artin dans $\mathbb{C}[[u]]\left(M^{(\mu)}\right)$ (cf. [9]), il existe des séries $\widetilde{\mathcal{A}}(u)$ et $\widetilde{Q}_{i}(u)$, avec $i=1, \ldots, m$, qui vérifient la relation

$$
\widetilde{\mathcal{A}}(u)^{l}+p_{1}(u) \widetilde{\mathcal{A}}(u)^{l-1}+\ldots+p_{l-1}(u) \widetilde{\mathcal{A}}(u)+p_{l}(u)=\sum_{i=1}^{m} \widetilde{Q}_{i}(u) a_{i}(u) .
$$

En regroupant (18.3) et (18.4), on obtient l'existence de séries $\alpha_{i}(u)$, pour $i=1, \ldots, l-2$, de $\mathbb{C}[[u]]\left(M^{(\mu)}\right)$ telles que l'on ait

$$
\begin{aligned}
(\mathcal{A}(u)-\widetilde{\mathcal{A}}(u))\left(\mathcal{A}(u)^{l-1}+\alpha_{1}(u) \mathcal{A}(u)^{l-2}+\ldots\right. \\
\left.+\alpha_{l-2}(u) \mathcal{A}(u)+p_{l-1}(u)\right) \in \mathcal{R} .
\end{aligned}
$$

Cas $l \geq 2$. Si on a $\mathcal{A}(u)^{l-1}+\alpha_{1}(u) \mathcal{A}(u)^{l-2}+\ldots+\alpha_{l-2}(u) \mathcal{A}(u)+p_{l-1}(u)$ $\in \mathcal{R}$, alors on obtient clairement un polynôme de degré $l-1$ qui appartient à $E$. Ceci est impossible par minimalité de $l$. Comme $\mathcal{R}$ est un idéal premier, on a donc $\mathcal{A}(u)-\widetilde{\mathcal{A}}(u) \in \mathcal{R}$. Ainsi il existe une série $\widetilde{\mathcal{A}}$ de $\mathbb{C}[[u]]\left(M^{(\mu)}\right)$ telle que l'on ait

$$
\widetilde{\mathcal{A}} \circ F \circ H=\mathcal{A} \circ F \circ H .
$$

Comme le jacobien de l'application $H$ est non nul, on a aussi $\widetilde{\mathcal{A}} \circ F=\mathcal{A} \circ F$.

Cas $l=1$. De (18.5), on tire directement $\mathcal{A}(u)-\widetilde{\mathcal{A}}(u) \in \mathcal{R}$. On termine comme dans le cas précédent.

Remarques. Lorsque l'application $F$ est de rang générique $r>1$ non maximal, sous quelles hypothèses a-t-on encore $(* *)$ ? Dans le cas analytique cette question est résolue dans [6]. Dans le cas Gevrey, le problème reste ouvert. On peut cependant remarquer que la démonstration précédente reste valable lorsque l'on a encore (18.1), c'est-à-dire lorsque l'idéal engendré par les composantes de l'application $G$, obtenue après transformation de $F$ comme dans le lemme 17, est de codimension finie. 
Remerciements. Je tiens à remercier chaleureusement Arkadiusz Płoski pour les discussions fructueuses que j'ai eues avec lui sur [14]. Enfin, j'exprime aussi toute ma gratitude à Edward Bierstone pour les nombreux échanges qu'il m'a accordés et pour son invitation à l'Université de Toronto où une partie de ce travail a été réalisée.

\section{Références}

[1] J. M. Aroca, H. Hironaka and J. L. Vicente, The theory of the maximal contact, Memorias de Matemática del Instituto "Jorge Juan" 29, 1975.

[2] J. Chaumat et A.-M. Chollet, Caractérisation des anneaux noethériens de séries formelles à croissance contrôlée. Application à la synthèse spectrale, Publ. Mat. 41 (1997), 545-561.

[3] -, 一, Théorèmes de préparation dans des classes ultra-différentiables, Math. Z. 226 (1997), 631-679.

[4] —, - On composite formal power series, Trans. Amer. Math. Soc. 353 (2001), 1691-1703.

[5] P. M. Eakin and G. A. Harris, When $\Phi(f)$ convergent implies $f$ is convergent, Math. Ann. 229 (1977), 201-210.

[6] A. M. Gabrielov, Formal relations between analytic functions, Math. USSR-Izv. 7 (1973), 1056-1088.

[7] E. Maillet, Sur les séries divergentes et les équations différentielles, Ann. Sci. École Norm. Sup. 3 (1903), 487-518.

[8] B. Malgrange, Ideals of Differentiable Functions, Oxford Univ. Press, 1966.

[9] A. Mouze, Un théorème d'Artin pour des anneaux de séries formelles à croissance contrôlée, C. R. Acad. Sci. Paris 330 (2000), 15-20.

[10] —, Division dans l'anneau des séries formelles à croissance contrôlée. Applications, Studia Math. 144 (2001), 63-93.

[11] - Sur la composition de certaines séries formelles, Ann. Scuola Norm. Sup. Pisa (2002), à paraître.

[12] —, Anneaux de séries formelles à croissance contrôlée, thèse, Univ. de Lille, 2000.

[13] V. P. Palamodov, Multiplicity of holomorphic mappings, Functional Anal. Appl. 1 (1967), 218-226.

[14] A. Płoski, Sur les dérivations des anneaux des séries convergentes, in: Lecture Notes in Math. 1039, Springer, Berlin, 1983, 389-393.

[15] J. C. Tougeron, Idéaux de fonctions différentiables, Springer, Berlin, 1972.

Laboratoire de Mathématiques, UMR 8524

Université des Sciences et Technologies de Lille

59650 Villeneuve d'Ascq Cedex, France

E-mail: mouze@agat.univ-lille1.fr 